\title{
LA SENSIBILITÉ TACTILE, OU LE COMPAS DE WEBER Nélia Dias
}

\author{
C.N.R.S. Editions | « Hermès, La Revue »
}

2016/1 $n^{\circ} 74 \mid$ pages 139 à 142

ISSN 0767-9513

ISBN 9782271090171

Article disponible en ligne à l'adresse :

http://www.cairn.info/revue-hermes-la-revue-2016-1-page-139.htm

\section{Pour citer cet article :}

Nélia Dias, "La sensibilité tactile, ou le compas de Weber », Hermès, La Revue 2016/1 (n 74), p. 139-142.

Distribution électronique Cairn.info pour C.N.R.S. Editions.

(C) C.N.R.S. Editions. Tous droits réservés pour tous pays.

La reproduction ou représentation de cet article, notamment par photocopie, n'est autorisée que dans les limites des conditions générales d'utilisation du site ou, le cas échéant, des conditions générales de la licence souscrite par votre établissement. Toute autre reproduction ou représentation, en tout ou partie, sous quelque forme et de quelque manière que ce soit, est interdite sauf accord préalable et écrit de l'éditeur, en dehors des cas prévus par la législation en vigueur en France. Il est précisé que son stockage dans une base de données est également interdit. 


\section{La sensibilité tactile, ou le compas de Weber}

«Toute la technique de l'esthésiométrie pourrait tenir dans ce principe : ne pas oublier que, lorsqu'on mesure la sensibilité tactile, on fait de la psychologie.» (Binet, 1902) C'est avec cette phrase que le psychologue français Alfred Binet (1857-1911) termine son article intitulé «La mesure de la sensibilité», article qui était l'aboutissement de toute une série de travaux publiés dans L’Année psychologique consacrés à la mesure en psychologie, à l'esthésiomètre (instrument de mensuration de la sensibilité tactile) et à ses procédures méthodologiques. Par ailleurs, Victor Henri avait publié en 1895 dans cette même revue sur la localisation des sensations tactiles, sujet de sa thèse de doctorat soutenue en 1897 (Nicolas, 1994). Pour quelles raisons les psychologues de la fin du XIx ${ }^{e}$ siècle en France ont-ils examiné tant les techniques de mesure de la sensibilité tactile que les types de phénomènes (sensation tactile et sensibilité tactile d'une part, sensibilité tactile et sensibilité de l'autre) susceptibles d'être mesurés?

C'est dans les années 1830 avec l'émergence de la psychophysique que l'approche quantitative de la physiologie des sens - notamment l'étude de l'acuité tactile et de la sensation tactile - a pris un essor considérable. Des décennies plus tard, ce sera le tour des anthropologues - dans leur recherche des différences culturelles et physiologiques en fonction de l'appartenance ethnique d'examiner les variations de la sensibilité tactile au sein du genre humain et de mesurer la sensation tactile à l'aide, entre autres, du compas de Weber (Dias, 2007). Ce compas conçu par Ernst Heinrich Weber (1795-1878) pour établir des relations quantitatives entre l'intensité du stimulus et la sensation, dans le cadre de ses recherches psychophysiques, va se répandre largement, tout au long du $\mathrm{XIX}^{\mathrm{e}}$ siècle, dans la pratique médicale et en anthropologie. C'est dans ce contexte qu'on peut situer les démarches de Binet démontrant que l'étude et la mesure de la sensibilité tactile ne relevaient pas de la physiologie mais bel et bien de la psychologie. En mettant en évidence d'une part les relations entre la finesse du toucher et le degré de l'intelligence, et de l'autre la nécessaire prise en compte des facteurs d'ordre mental (parmi lesquels l'attention et la mémoire) dans la mesure de la sensibilité tactile (Binet, 1899a), le psychologue français a été amené à critiquer la fiabilité des instruments de mesure, notamment le compas de Weber.

\section{La question de la mesure}

Weber avait souligné la façon dont deux sensations sont perçues différemment en fonction de l'intensité de la stimulation. Il entendait vérifier que, pour chaque ordre de sensation et donc pour les sensations tactiles, il existe un rapport constant entre la grandeur de l'excitant (dans ce cas le poids des pointes du compas) et celle dont il faut l'augmenter pour que la différence soit sentie - la plus petite grandeur dont il faut accroitre ou diminuer l'excitant d'une sensation pour que la variation soit sentie définissant le seuil différentiel. Ce fut Gustav Theodor Fechner qui donna une formulation mathématique de la relation entre sensation et stimulation et la nomma la « loi de Weber "; cette loi établit que l'ampleur avec laquelle est ressenti un stimulus est proportionnelle au logarithme de son intensité physique (Boring, 1929, p. 280-281).

Weber avait constaté que certaines des régions du corps humain - la pointe de la langue, les lèvres, l'extrémité des doigts et la plante des pieds - étaient plus richement innervées que d'autres et devaient par conséquent 
réagir avec plus d'intensité à des excitations provoquées. Il avait identifié plus de quarante régions du corps, désignées sous le nom de "cercles de la sensation», établies en fonction des endroits précis du corps où la double sensation causée par les deux pointes du compas se commue en sensation unique comme si elle avait été provoquée par une seule pointe. Suivant l'écartement des branches du compas, le sujet d'expérimentation accusait la sensation de deux pointes ou d'une seule. Il était, par conséquent, possible d'établir, pour chaque région du corps humain et pour chaque individu, jusqu'à quel degré les pointes du compas pouvaient être rapprochées avant que deux sensations distinctes ne se transforment en une unique sensation.

La manipulation du compas requérait un protocole expérimental spécifique : le sujet d'expérimentation devait garder les yeux fermés pendant la durée du test. Il était, en effet, indispensable d'éviter que les sensations tactiles à mesurer puissent être influencées par la vision des pointes du compas ou de la région du corps soumise à l'expérience. Le risque était alors que les «sensations visuelles» interfèrent avec les sensations tactiles et perturbent la portée de l'expérience.

Dans ses articles, Binet (1902, p. 85) s'attelle à critiquer aussi bien la loi Weber-Fechner - qualifiée de «conception mathématique de la sensibilité» (tout en admettant qu'elle «mérite aujourd'hui encore notre admiration ») - que les techniques de mesure à l'aide du compas de Weber et les résultats obtenus. Trois aspects sont à souligner : premièrement, la distinction établie par le psychologue français entre mesure de la sensibilité (qui porte sur l'exactitude d'un jugement) et mesure des sensations (qui porte sur l'intensité des sensations) (Ibid.). Dans ce dernier cas, la «mesure porte sur l'excitant» (poids, son, rayon de lumière) et par conséquent «on ne fait point d'analyse mentale» (Ibid., p. 82). Deuxièmement, tout en louant la topographie de la sensibilité tactile élaborée par Weber, Binet émet cependant des réticences concernant l'épreuve de discrimination de deux pointes établie à l'aide du compas. Comme il l'écrit : «Il n'est pas aussi facile qu'on le croit d'appliquer deux pointes dans des conditions irréprochables, sans exercer de petite modification qui tantôt facilite, tantôt obscurcit la dissociation des deux contacts simultanés par l'intelligence du sujet.» (Binet, 1900a, p. 232) Autrement dit, la sensation de deux pointes dérivait de l'interprétation donnée par le sujet d'expérimentation. Cet aspect est étroitement lié au suivant, à savoir la critique des protocoles méthodologiques adoptés par Weber. Selon Binet (1902), «l'erreur de Weber» réside dans le fait de ne pas avoir tenu compte du déroulement des expériences, du choix des sujets d'expérimentation, de la possibilité que le nombre de mesures effectuées ait pu affecter l'attention du sujet d'expérimentation et de la manière dont l'expérience devait être expliquée aux sujets. En laissant de côté la question de la manière d'interpréter les réponses des sujets d'expérimentation, Weber aurait réduit ces derniers «à l'état d'automate» (p. 122).

\section{Un instrument qui légitime la normalisation?}

S’il revient à Binet le mérite d'avoir systématisé la distinction entre sensation et perception, et d'avoir précisé le contenu de la notion de sensibilité (et par là ce qui est en jeu dans la mesure de la sensibilité), il n'en reste pas moins que ses critiques à l'égard de Weber semblent trop sévères. Weber avait mis l'accent sur le rôle susceptible d'être joué par l'expérience personnelle et les habitudes du sujet dans sa capacité à la discrimination tactile. Le poids conjugué de ces deux facteurs pouvait contribuer à diminuer le nombre des «cercles de la sensation", ces zones où la double sensation des deux pointes vient à se transformer en sensation unique. 
C'est parce que Weber avait pris en ligne de compte des facteurs d'ordre psychologique et social tels que l'expérience et l'habitude - en admettant qu'elles diminuaient le nombre de cercles nécessaires pour que l'intervalle fût perçu entre deux points du corps - dans l'évaluation des capacités sensorielles qu'il sera rangé par Théodule Ribot (1879, p. 105) parmi les partisans de la théorie dite "empirique». Weber ne s'était pas contenté de compter l'expérience passée du sujet et les habitudes formées par lui au nombre des facteurs susceptibles d'influer sur les réponses de ce sujet aux stimulations cutanées. Il y avait également inclus la mémoire. Le souvenir des sensations ressenties pouvait, selon lui, intervenir dans des sensations ultérieurement éprouvées. En d'autres termes, des données d'ordre culturel, liées aux spécificités des modes de vie (les habitudes acquises au travers des exercices quotidiens) et des facteurs psychologiques (l'attention, la mémoire) affectaient le pouvoir de discrimination tactile et modifiaient les seuils différentiels.

La sensibilité tactile, mesurée grâce au compas, permettait de conférer une légitimité scientifique aux pratiques de normalisation à l'œuvre à cette époque. Ce n'est pas un hasard, comme l'a noté Michel Foucault (1975, p. 302), si c'est au moment de l'émergence de la psychologie et de «l'apparition de ces professionnels de

\section{RÉFÉRENCES B I B LIOGR A PHIQUES}

Binet, A., «La mesure en psychologie individuelle», Revue philosophique, $1898, \mathrm{n}^{\circ} 46$, p. 113-123.

Binet, A., "Attention et adaptation», L'Année psychologique, vol. $6, \mathrm{n}^{\circ} 1,1899 \mathrm{a}$, p. 248-404.

Binet, A., "Recherches sur la sensibilité tactile pendant l'état de distraction", L’Année psychologique, vol. 6, $\mathrm{n}^{\circ} 1,1899 \mathrm{~b}$, p. 405-440. la discipline, de la normalité et de l'assujettissement», que Weber, pour mesurer les sensations, a commencé à «manipuler son petit compas». Et Foucault de souligner la façon dont «l'estimation quantitative des réponses sensorielles et la mesure d'un seuil différentiel» faisaient partie intégrante "d'un nouveau type de contrôle - à la fois connaissance et pouvoir - sur les individus» (Ibid.). De plus, connaître de manière la plus détaillée possible les parties du corps les plus sensibles permettait d'aborder la question de la douleur, autrement dit de la sensibilité à la douleur. Les applications de ce type de dispositif en matière de gestion des comportements socialement déviants tant dans la métropole que dans les colonies n'étaient pas anodines.

On peut comprendre dès lors les raisons pour lesquelles l'anthropologie et la psychologie (Staum, 2007) ont pu partager, à une époque, des préoccupations théoriques concernant la quantification de phénomènes à la fois mentaux et physiologiques (la sensibilité, l'intelligence, l'attention, la respiration, la circulation artérielle) et des pratiques de mesure, au rang desquelles le compas de Weber.

Nélia Dias

ISCTE - Instituto universitário de Lisboa CRIA - Centre de recherches en anthropologie

Binet, A., «Un nouvel esthésiomètre», L'Année psychologique, vol. 7, n 1, 1900a, p. 231-239.

Binet, A., «Technique de l'esthésiométrie», L’Année psychologique, vol. 7, $\mathrm{n}^{\circ} 1,1900$ b, p. 240-248.

Binet, A., «La mesure de la sensibilité», L’Année psychologique, vol. 9, n 1, 1902, p. 79-128.

Boring, E. G., A History of Experimental Psychology, New York, Appleton, 1929. 
DiAs, N., «Une pointe ou deux? Le destin anthropologique du compas de Weber», Terrain, n 49, 2007, p. 51-62.

Foucault, M., Surveiller et Punir, Paris, Gallimard, 1975.

HENRI, V., «Recherches sur la localisation des sensations tactiles", L’Année psychologique, vol. 2, nº 1, 1895, p. 168-192.

Henri, V., «Revue générale sur le sens du lieu de la peau», L'Année psychologique, vol.2, $\mathrm{n}^{\circ} 1,1895$, p. 295-362.

Nicolas, S., "Qui était Victor Henri?», L’Année psychologique, n 94,1994, p. 385-402.
Riвot, T., La Psychologie allemande contemporaine, Paris, Baillière, 1879.

Staum, M., «Ribot, Binet, and the Emergence from the Anthropological Shadow", Journal of the History of the Behavioral Sciences, vol. 43, $\mathrm{n}^{\circ}$ 1, 2007, p. 1-18.

Weber, E. H., On the Tactile Senses, édité et traduit par Helen E. Ross et David J. Murray, Erlbaum, Taylor and Francis, 1996 [2éd.]. 